\title{
Production of cadmium sulphide quantum dot nanoparticles using tomato hairy root tissue culture: ultrastructural effects and localization of cadmium
}

\author{
K.J. Levick, ${ }^{*}$ M. Stevens-Kalceff,* H. Zhao, ${ }^{* *}$ and P.M. Doran** \\ * School of Physics and Electron Microscope Unit, University of New South Wales, Sydney, NSW \\ 2052, Australia \\ ** School of Biotechnology and Biomolecular Sciences, University of New South Wales, Sydney, \\ NSW 2052, Australia
}

Quantum dots are nanocrystal particles with unique electronic and optical properties. New applications of quantum dots are being developed in areas such as medical imaging, diagnostics, targeted therapies and microelectronics.

Commercial expansion of quantum dot technology is limited by difficulties with mass production. The functional properties of quantum dots depend directly on quantum confinement effects, therefore close control must be exercised over the particle size (generally, particles 2-20nm in size are required). Quantum dots are most commonly produced using chemical methods involving crystal nucleation in aqueous or organic solvents. However, it is difficult using this approach to produce monodispersed nanocrystals while preventing particle instability and aggregation. Improved techniques for producing high quality quantum dots cheaply, reproducibly and in large quantities are needed.

A potential solution to these problems is the biological production of quantum dots. As microscopic reaction chambers, cells provide the space confinement conditions to generate nanoparticles of restricted size. However, cadmium is a highly toxic heavy metal which has been found to change root morphology, damage cell structures and disturb water balance and mineral nutrition, among other effects [1]. When faced with an excess of heavy metals in the environment plants may either attempt to avoid the stress by restricting the uptake of metals through the root or attempt to minimize the toxic effects of the metal ions in the cell. Plants detoxify heavy metals such as cadmium by complexing them with sulphur and then capping the complex with chelating peptides known as phytochelatins [2].

It has been found that hairy root cultures of tomato (Lycopersicon esculentum) produce cadmium sulphide nanoparticles and phytochelatins in response to exposure to $\mathrm{CdSO}_{4}[3,4]$. Exposure to $\mathrm{CdSO}_{4}$ has also been found to have a significant and detrimental effect on the ultrastructure of the hairy root culture [5].

In this study the effect on the tomato hairy root culture of exposure to cadmium was investigated. The effect of the cadmium increased over time (1-14 days). The morphology of the root was examined using scanning electron microscopy and the ultrastructure was examined using transmission electron microscopy. Exposure to cadmium caused significant ultrastructural damage to the cell. Electron dense granules were observed in the cytoplasm of the treated cells. The localization of the cadmium was also investigated using energy dispersive x-ray microanalysis. The cadmium was found to be concentrated in specific areas (eg. the electron dense granules). 
References

[1] M.N.V. Prasad, Environ. Exp. Bot 35 (1995) 525.

[2] C.T. Dameron and D.R. Winge, Trends in Biotech. 8 (1990) 3.

[3] H. Zhao et al Abstracts of the Biochemical Engineering XIV Conference (2005) p68.

[4] H. Zhao et al Materials Research Society Meeting: Symposium on Quantum-Confined Semiconductor Nanostructures - Fabrication, Physical Properties, and Applications (2005).

[5] K.J. Levick et al ACMM19, Microscopy Solutions. ed. P Munroe et al ISBN 0-9580408-4-2 AMMS. Inc., Sydney (2006) p165.

[6] This work is funded by the Australian Research Council (ARC). J Norman is gratefully acknowledged for technical support.
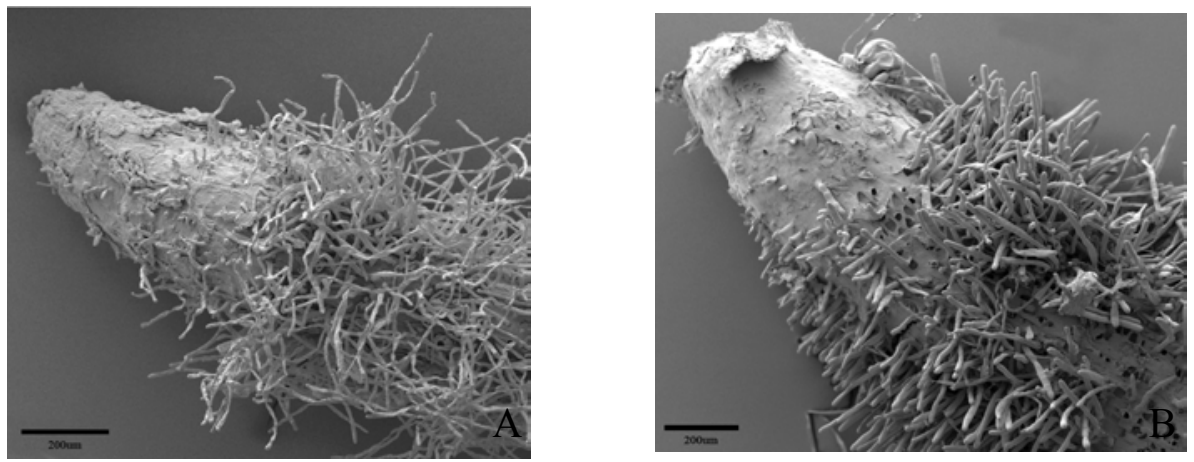

FIG. 1. A. Control root tip. B. Root tip after 14 days exposure to $100 \mu \mathrm{M} \mathrm{CdSO}_{4}$. Note reduction in length of root hairs and damage to root cap.
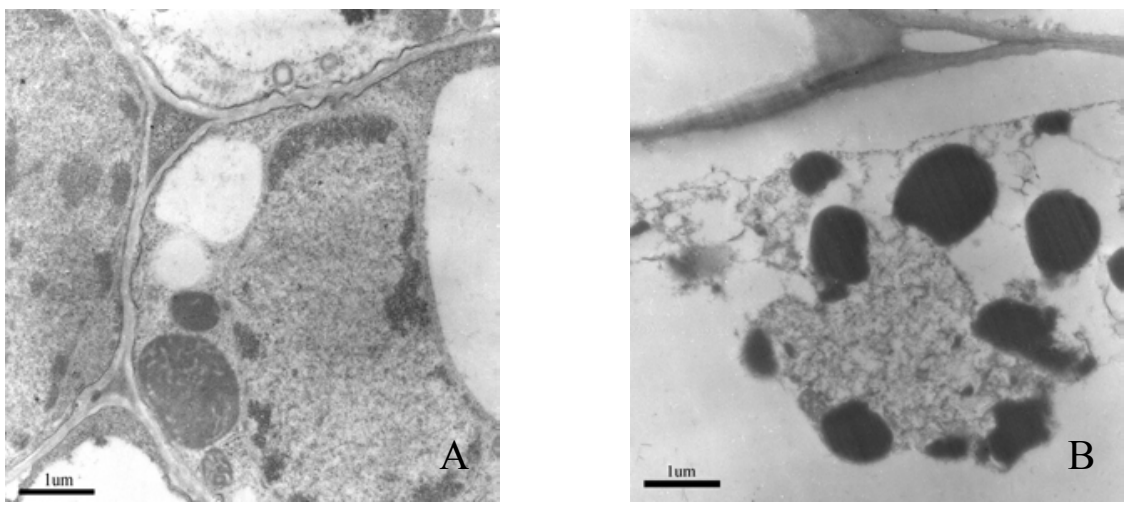

FIG. 1. A. Control stele cell. B. Stele cell after 14 days exposure to $100 \mu \mathrm{M} \mathrm{CdSO}_{4}$. Note damage to cytoplasm and accumulation of electron dense granules. 